\title{
Development of new correlations for annular flow
}

\author{
C. Berna ${ }^{1}$, A. Escrivá ${ }^{1}$, J. L. Muñoz-Cobo ${ }^{1}$ \& L. E. Herranz ${ }^{2}$ \\ ${ }^{1}$ Instituto de Ingeniería Energética, Universitat Politècnica de València, \\ Spain \\ ${ }^{2}$ CIEMAT, Spain
}

\begin{abstract}
Annular two-phase flow has been vastly investigated due to its large and deep involvement in a large amount of industrial processes, for instance, petroleum, chemical, civil and nuclear industries, and particularly in boiling and condensing heat transfer equipment. Annular flow is characterized by a thin liquid film flowing on the pipe wall and a high velocity gas core flowing in its centre, which normally carries liquid droplets.

This work reviews most of the recent literature on the matter, with emphasis in all important variables which control the annular flow. This work focuses on the processes occurring in the liquid-gas interface that cause droplet entrainment, addressing also the characterization of these entrained droplets. This paper shows the existing scattering when key variables expressions are compared to each other and it highlights the gaps of knowledge still existing.

Additionally, based on some of the open experimental data, alternate equations for liquid film thickness, wave celerity, wave frequency, droplet sizes and total droplet amount, have been derived for annular flow. The performance of the correlations derived is shown to enhance the existing predictability. It is noteworthy that the correlations derived are based on non-dimensional numbers, which makes them suitable for their application to a broader set of scenarios (i.e., fluids, geometry and so on).

Keywords: annular flow, entrainment, gas-liquid interface, film thickness, wave celerity and frequency, droplet size and velocity, entrained fraction.
\end{abstract}

\section{Introduction}

The study of two phase flows, and particularly annular flow, is important due to its relevance in many industrial applications: petroleum, chemical, civil and 
nuclear industries. Annular flow is encountered in many processes; for instance, channel flow during steam generation processes, nuclear reactors and other power plants, heating and refrigeration equipment, such as, heat exchangers and condensers, gas-liquid mixers and gas-liquid separators, transportation of natural gas and crude oil, etc.

The annular flow has been the subject of many theoretical studies, for both horizontal and vertical pipes. When describing the annular flow, the scenario can be divided in three major features: the waves at the gas-liquid interface; the droplet entrainment into the gas core; and the deposition of a fraction of these entrained droplets. The processes that take place on the gas-liquid interface are at dynamic equilibrium with continuous exchange of mass between the liquid film and the gas stream, when this mass balance remains constant in the gas core, the fully developed annular flow is achieved.

A huge number of papers have been published previously on the issue addressed in this paper. Those papers with a major impact on the area will be properly referenced in next sections, but this paper also presents a new set of correlations for these three major features of the annular flow.

\section{Liquid film and wave characteristics}

Two different types of waves are considered that coexist on the liquid film surface: long-length disturbance waves (DW), with amplitude several times higher than the average liquid film thickness, and small-scaled ripple waves (RW). At very low liquid flow rates the RW dominate the two-phase interface, but above a critical liquid flow rate the DW appear. Although both types of waves coexist over the full range of annular flow, from this point on the DW dominate the process $[1,2]$. Consequently, only the DW is studied here.

An adequate characterization of these waves is important for entrainment modeling, because it deals with the prediction of the conditions at which waves are formed and become unstable. A complete description of their characteristics involves obtaining: celerity, wavelength, amplitude, shape, etc., see fig. 1.

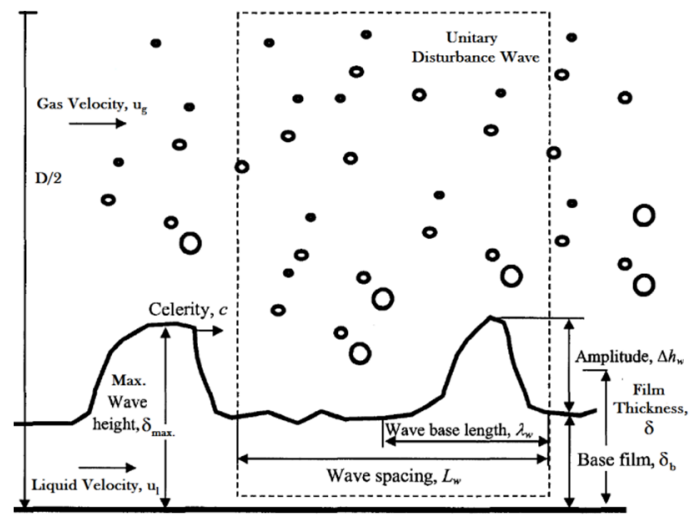

Figure 1: Schematic view of a unit DW. 


\subsection{Entrainment inception velocity and onset of entrainment Reynold number}

Previously to the characterization of the wave variables, it is needed to determine the conditions above which the entrainment process takes place. For this purpose, the entrainment inception velocity and the onset of entrainment Reynolds number have to be defined.

Low relative velocities between liquid and gas phases produce a smooth interface. As this difference in velocity increases, the interface covers with waves and, from a certain point, part of the liquid is dragged by the gas phase (onset of entrainment). The critical condition for the start of the entrainment process mainly depends on liquid and gas stream velocities, see fig. 2 . This dependence on liquid velocity is usually presented by the Re $\mathrm{e}_{\mathrm{ffOE}}$ (liquid Reynolds number above which entrainment is possible) and the dependence on gas velocity can be presented from the Kutateladze number (Crowe [3], which depends on gas velocity).

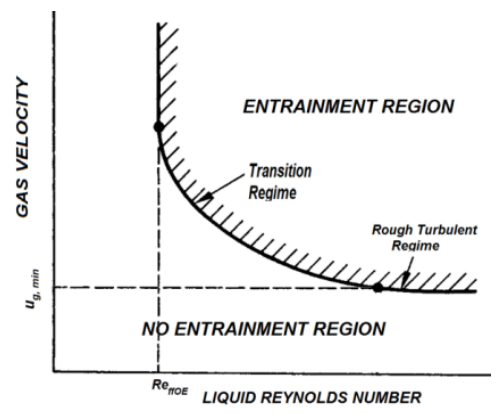

Figure 2: Schematic view of entrainment - no entrainment boundary.

For horizontal and vertical upward flows, which are the most widely studied due to its industrial importance, the onset of entrainment Reynolds number value is between 100 and 400 approximately, depending on the author. For instance, Ishii and Grolmes [4] suggest a value of 160, Okawa et al. [5] suggests a critical value of 320 from their experimental data, more elaborated expressions were proposed by other authors, like Owen and Hewitt [6] or Sawant et al. [7]. A value of 160 can be considered as a good approximation for $\mathrm{Re}_{\mathrm{ffOE}}$.

The original Kutateladze number criterion gives a constant entrainment inception velocity value (of the gas phase) depending only on gas and liquid properties. An improvement of the previous model is the one proposed by Crowe [3]. In that model, three regions are considered:

1. No entrainment zone. Reynolds numbers under $\mathrm{Re}_{\mathrm{ffoE}}$, where entrainment is not possible.

2. Rough turbulent zone. Liquid film Reynolds number exceeds a value of about 1500-1750 (value of $\operatorname{Re}_{1}=1635$ typically found in the literature), the liquid film flow becomes completely rough-turbulent and the entrainment inception velocity has a constant value $(\mathrm{Ku}=3.2)$. 


$$
K u^{2}=\frac{\rho_{g} u_{g}^{2}}{\sqrt{\sigma g \rho_{l}}} \geq 10.24
$$

3. Transition zone. Reynolds number between these two values, the entrainment inception velocity has not a constant value. At the lower liquid Reynolds side of the regime, the critical Kutateladze number is $\mathrm{Ku} \approx 7.5$. Using as a first approximation a value of 200 for the onset of entrainment Reynolds number, and the value of 1635 for the beginning of the rough turbulent region; then the transition regime covers the liquid film Reynolds numbers $160<\mathrm{Re}_{1}<1635$. So, considering as linear the variation in that transition region,

$$
K u^{2}=\frac{\rho_{g} u_{g}^{2}}{\sqrt{\sigma g \rho_{l}}} \geq 61.241-0.0312 \operatorname{Re}_{l}
$$

\subsection{Liquid film thickness}

Liquid film thickness is defined as the distance from the pipe wall to a "mean height" of the waves produced into the gas-liquid interface, see fig.1. Along the last decades many works have been concentrated on this variable, for instance the ones of Asali, Ambrosini, Fukano (Fukano and Furukawa [8]), Okawa et al. [5] or Ishii and Grolmes [4], Tatterson et al. [9] among others.

A lot experiments have been carried out in order to determine this magnitude. In the present work, we have analyzed the following experimental data series: Tatterson et al. [9], Paras and Karabelas [10], Schubring's [11] and Alamu's [12] and from them we have developed a new correlation to determine this magnitude.

$$
\frac{\delta}{D}=7.165 \cdot \operatorname{Re}_{g}^{-1.07} \operatorname{Re}_{l}^{0.48}\left(\frac{F r_{g}}{F r_{l}}\right)^{0.24}
$$

where, for the gas and liquid phases, the Reynolds and Froude numbers are defined by:

$$
\operatorname{Re}_{g}=\frac{\rho_{g} J_{g} D}{\mu_{g}} ; \operatorname{Re}_{l}=\frac{\rho_{l} J_{l} D}{\mu_{l}} ; F r_{g}=\frac{J_{g}}{\sqrt{g D}} ; F r_{l}=\frac{J_{l}}{\sqrt{g D}}
$$

all these dimensionless numbers are defined in terms of superficial velocities.

The fitting of the new correlation with the experimental data analyzed is shown in fig. 3. The new correlation produces a reasonable fitting for all experimental data, as they almost collapse to the correlation's line (almost all data located between the error lines of $\pm 25 \%$, Pearson product-moment coefficient of $\left.\mathrm{R}^{2}=0.902\right)$.

\subsection{Wave celerity}

The wave celerity or just "celerity", c, is the wave displacement velocity. This magnitude has been widely studied, highlighting the works of Kumar (Mantilla, [13] or Al-Shakhi et al. [14] among others).

To determine this variable we have analyzed the following experimental data series: Schubring's [11], Alamu's [12] and Mantilla's [13]. A large number of 


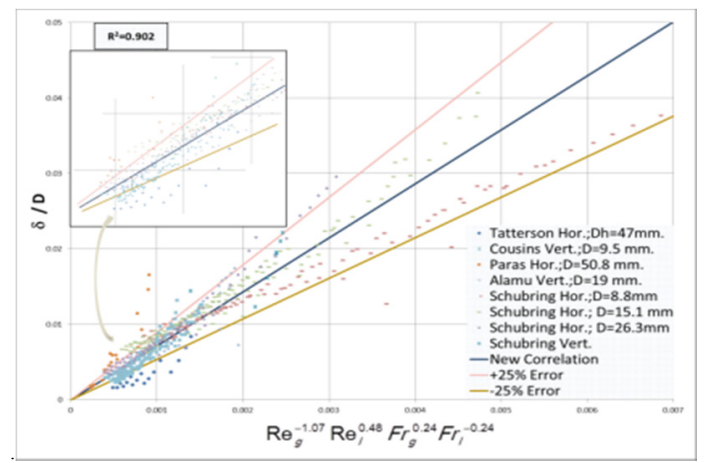

Figure 3: Fitting of the experimental data with the new film thickness correlation.

adjustments have been made in order to correlate wave celerity with physical properties and conditions at which the experiments have been carried out. To normalize wave celerity, various possibilities have been tested, such as: dividing by gas superficial velocity, the square root of gas and liquid velocities product, etc., but the final choice is the one shown below. Then, the new correlation is

$$
\frac{c}{\frac{\sqrt{\rho_{g}} J_{g}+\sqrt{\rho_{l}} J_{l}}{\sqrt{\rho_{g}}+\sqrt{\rho_{l}}}}=\operatorname{Re}_{g}^{-0.38} \mathrm{Re}_{l}^{0.16} C_{W}^{-0.13}
$$

being the gas and liquid Reynolds numbers as defined in eqn. (4). Whereas the surface tension factor $\left(\mathrm{C}_{\mathrm{W}}\right)$ is defined as originally by Ishii and Grolmes [4]:

$$
\begin{array}{lll}
C_{W}=0.028 N_{\mu}^{-4 / 5} & \text { for } & N_{\mu} \leq 1 / 15 ;
\end{array} \quad N_{\mu}=\mu_{l} /\left(\rho_{l} \sigma \sqrt{\frac{\sigma}{g \Delta \rho}}\right)^{1 / 2}
$$

The fitting of the proposed new correlation with the experimental data is presented in fig. 4. The new correlation produces a reasonable fitting of all experimental data, as they collapse almost all data to the correlation's line (almost all data located between the error lines of $\pm 25 \%$, Pearson productmoment coefficient of $\mathrm{R}^{2}=0.885$ ).

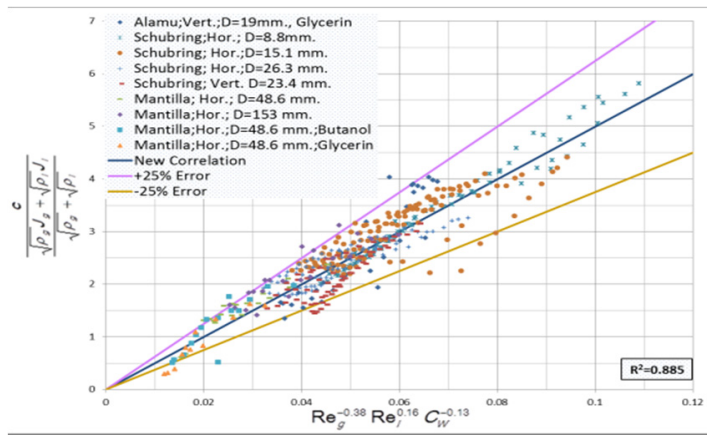

Figure 4: Fitting of the experimental data with the new wave celerity correlation. 


\subsection{Wave frequency}

The wave frequency is the number or waves per unit of time. The experimental series analyzed to study the wave frequency are the same ones that have been used to correlate the wave celerity in the previous section [11-13]. The same procedure than in the previous section has been followed. Finally, the new correlation proposed is as follows,

$$
S t_{g l}=\operatorname{Re}_{g}^{0.53} \operatorname{Re}_{l}^{-0.48} E o^{0.27}\left(\frac{\rho_{g}}{\rho_{l}}\right)^{0.14} C_{W}^{0.68}
$$

where $\mathrm{St}_{\mathrm{gl}}$ (gas-liquid Strouhal number) and Eo (Eötvös number) are defined as:

$$
S t_{g l}=\frac{v D}{\sqrt{J_{g} \cdot J_{l}}} ; E o=\frac{g D^{2}\left(\rho_{l}-\rho_{g}\right)}{\sigma}
$$

the gas and liquid Reynolds numbers defined as in eqn (4) and $C_{W}$ as in eqn (6).

The fitting of the proposed new correlation with the experimental data shown in the previous paragraphs is presented in fig. 5. The new correlation does not produce a quite good fitting of the experimental data (almost all data located between the error lines but of $\pm 50 \%$, Pearson product-moment coefficient of $\mathrm{R}^{2}=0.683$ ).

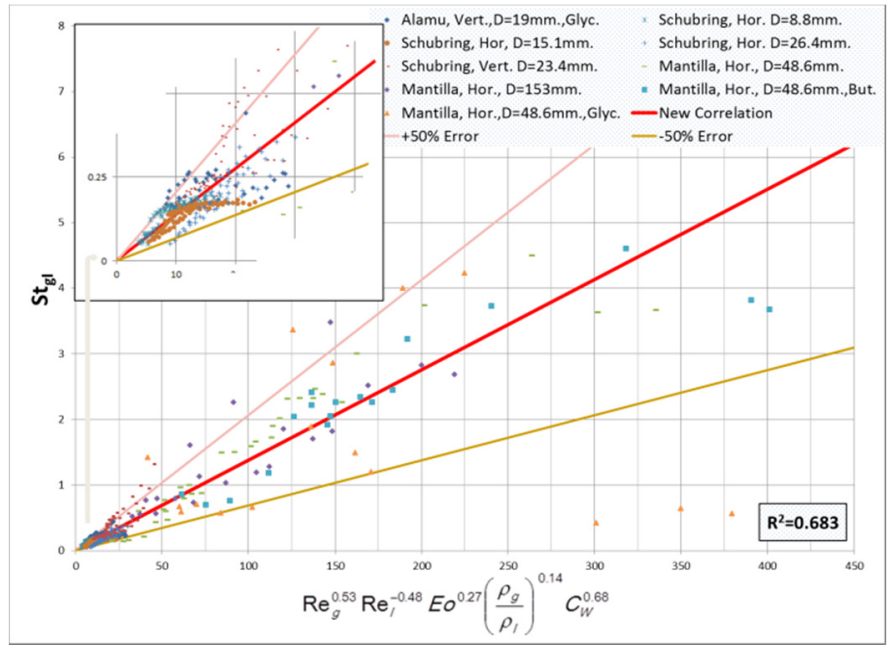

Figure 5: Fitting of the experimental data with the new wave frequency correlation.

\section{Characterization of the entrained droplets}

Once the liquid phase has been described, the final step is the determination of the key variables of the entrained droplets. That is, size, velocity and total amount of droplets. 


\subsection{Droplet sizes}

The determination of the initial droplet sizes are determined by the mechanism from which have been generated, such as shearing off of roll-wave crests in the case of annular flow, or primary atomization in the case of sprays formation from a liquid jet or sheet. But, in addition to the formation mechanisms, when these droplets are surrounded by a gas which is moving at a high relative velocity, the aerodynamic forces will cause the deformation and fragmentation of these droplets. Then the droplets with a diameter larger than the maximum stable size begin to oscillate, which finally results in the rupture of the droplet. Detailed developments of the droplets breakup phenomenon can be found in several works, for instance, accurate descriptions are shown in Crowe [3] or Kolev [15]. Consequently, a distribution of smaller droplets is produced by the generation mechanisms.

The Weber number, which is the ratio between stress forces (trying to disrupt the droplets) and surface tension forces (trying to avoid droplet deformation) can be considered to determine droplet sizes. Based on experimental data it has been observed that for low viscosity liquids, We crit ranges from 5 to 20 (being the most widely used a value of 12) [15]. The $\mathrm{We}_{\text {crit }}$ is defined as,

$$
W e_{c r i t}=\frac{\rho_{g} u_{g}^{2} \phi_{d, \max }}{\sigma}=\text { CONSTANT }(5-20)
$$

Another way to estimate droplet sizes is just the direct correlation of experimental data as function of the scenario characteristics, flow conditions and fluid properties. The general form that present all this correlations is as follows:

$$
\frac{\phi_{v m}}{D}=C T E * W e_{g}^{A} \operatorname{Re}_{g}^{B} \operatorname{Re}_{l}^{C}\left(\frac{\rho_{g}}{\rho_{l}}\right)^{E}\left(\frac{\mu_{g}}{\mu_{l}}\right)^{F} C_{W}^{G}
$$

being the exponents and coefficients proposed by several researchers summarized in table 1. Added to these correlations, the ones developed here are also shown. In eqn (10) the gas and liquid Reynolds numbers are defined as in eqn (4), $C_{W}$ as in eqn (6) and the Weber number is defined as:

$$
W e_{g}=\frac{\rho_{g} J_{g}^{2} D}{\sigma}
$$

The experimental data used to develop these new correlations are: for horizontal flow, Paras and Karabelas [16], Simmons and Hanratty [17] and AlSarkhi and Hanratty [18] and secondly, the ones for vertical upward flow, Lopes [19], Fore and Duckler [20] and Fore et al. [21]. Highlight that a better fit has been appreciated if vertical upward and horizontal flows are separately correlated. Also comment that the pressure ratio of the new vertical flow correlation is considered as a correction due to the data adjustment at different pressures, while for horizontal flow no data were available for different pressures so this term could not have been captured.

The fitting of the proposed new correlations with the experimental data shown in the previous paragraphs are presented in figs 6 and 7. Regarding to the new correlation for horizontal flow say that this equation fits data much closer 
than the previous ones (almost all data located between the error lines of $\pm 25 \%$, Pearson product-moment coefficient of $\left.\mathrm{R}^{2}=0.902\right)$. Whereas the one for the vertical upward flow (almost all data located between the error lines of $\pm 25 \%$, Pearson product-moment coefficient of $\mathrm{R}^{2}=0.945$ ).

Table 1: Summary of the exponents and coefficients of droplet size correlations.

\begin{tabular}{|l|c|c|c|c|c|c|c|}
\hline Correlation & CTE & $\mathrm{A}$ & $\mathrm{B}$ & $\mathrm{C}$ & $\mathrm{E}$ & $\mathrm{F}$ & $\mathrm{G}$ \\
\hline Tatterson & 0.106 & $-1 / 2$ & $1 / 10$ & - & - & - & - \\
\hline Ishii & 0.0099 & -1 & $2 / 3$ & - & $-1 / 3$ & $2 / 3$ & - \\
\hline Kataoka & 0.028 & -1 & $2 / 3$ & $-1 / 6$ & $-1 / 3$ & $2 / 3$ & - \\
\hline Kocamustafao-gullari & 0.89 & $-3 / 5$ & $4 / 15$ & $-1 / 15$ & $4 / 15$ & $4 / 15$ & $-4 / 15$ \\
\hline Patruno & 0.007 & 0.75 & -1.97 & 0.2 & 1.23 & -4.47 & 5.18 \\
\hline New Horizontal & 2.634 & -0.23 & -0.54 & 0.13 & - & - & - \\
\hline New Vertical Upward & 0.11 & -0.68 & 0.33 & 0.11 & 0.31 & - & - \\
\hline
\end{tabular}

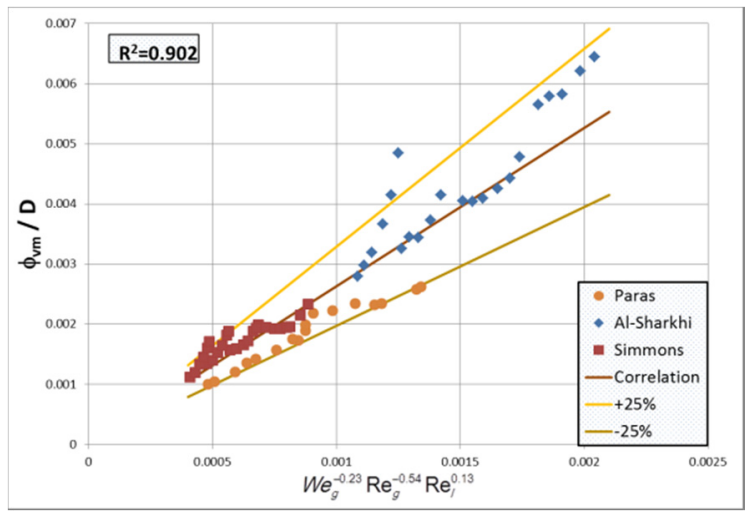

Figure 6: Fitting of the experimental data with the new droplet size correlation for horizontal flow.

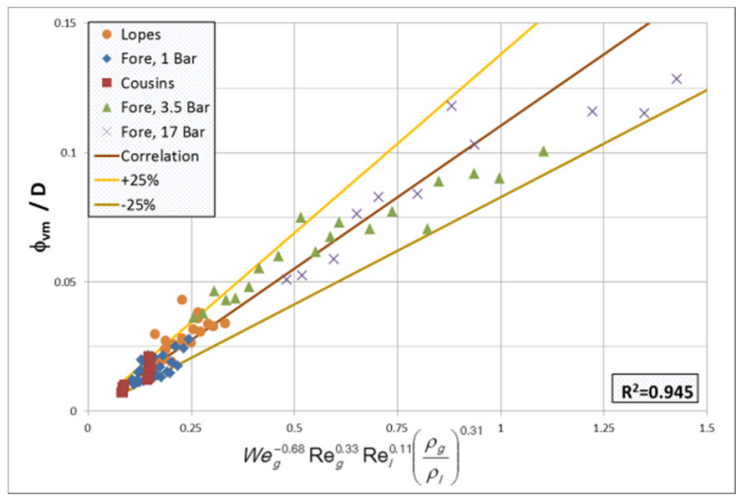

Figure 7: Fitting of the experimental data with the new droplet size correlation for vertical upward flow. 
Add that these new correlations are more accurate than critical Weber number criterion in both flow configurations, even though, if only the experimental gas velocity measurement is available this criterion provides a reasonable approximation.

\subsection{Droplet velocities}

Another key factor in the study of interactions between droplets and gaseous jets is the droplet velocity profile. The Stokes number is a very important dimensionless number when particles are suspended in a fluid flow, it is defined as the ratio of the particle momentum response time (in our case the droplet response time) over the flow system time. Mathematically:

$$
S t k=\frac{\tau_{d}}{\tau_{g}} ; \tau_{d}=\frac{\rho_{d} \phi_{d}^{2}}{18 \mu_{g}} ; \tau_{g}=\frac{D}{u_{g}}
$$

Then the relation between droplet velocity and gas velocity (slip ratio, $\mathrm{S}_{\mathrm{R}}$ ) is approximately equal to [3]:

$$
S_{R}=\frac{u_{d}}{u_{g}} \approx \frac{1}{1+S t k}
$$

Droplet velocity has been also estimated from experimental measurements $[20,22]$, the researchers propose values of $S_{R} \approx 0.8$, but they comment that there is a trend to travel at higher velocities for smaller droplets.

\subsection{The entrained fraction}

A crucial parameter in the analysis and modeling of annular flows is the fraction of liquid entrained as droplets in the gas core (entrained fraction), defined as:

$$
E=\frac{W_{l e}}{W_{l}}
$$

being $\mathrm{W}$ the mass flow rate, subscript le corresponds to the mass flow of droplets and 1 to the total mass flow rate of liquid. A good compilation of correlations are shown in Mantilla's PhD work [13], for instance the ones of Oliemans, Ishii and Mishima, Wallis, Paleev and Filippovich, etc. (the available experimental data covered not only water, but mixtures of water-glycerin and water-butanol, which provides a variation in the physical properties of the working fluids).

Lots of experiments have been carried out over the lasts decades in order to determine the entrained fraction. In the present work, we have analyzed the following experimental data series: Azzopardi et al.'s [23], Simmons and Hanratty's [17], Mantilla's [13] and Alamu's [12]. From these experimental data the next correlation has been developed:

$$
\frac{E}{1-E}=5.51 \times 10^{-7} \cdot W e_{g}^{2.68} \operatorname{Re}_{g}^{-2.62} \operatorname{Re}_{l}^{0.34}\left(\frac{\rho_{g}}{\rho_{l}}\right)^{-0.37}\left(\frac{\mu_{g}}{\mu_{l}}\right)^{-3.71} C_{W}^{4.24}
$$

the gas and liquid Reynolds numbers are defined as in eqn (4), $\mathrm{C}_{\mathrm{W}}$ as in eqn (6) and the Weber number as in eqn (11). 
Data fitting by this new correlation is presented in fig. 8. The data show a large scattering that prevents this correlation from having higher accuracy than data. Despite that, most data fall in between $\pm 50 \%$ being the Pearson productmoment correlation coefficient of $\mathrm{R}^{2}=0.666$.

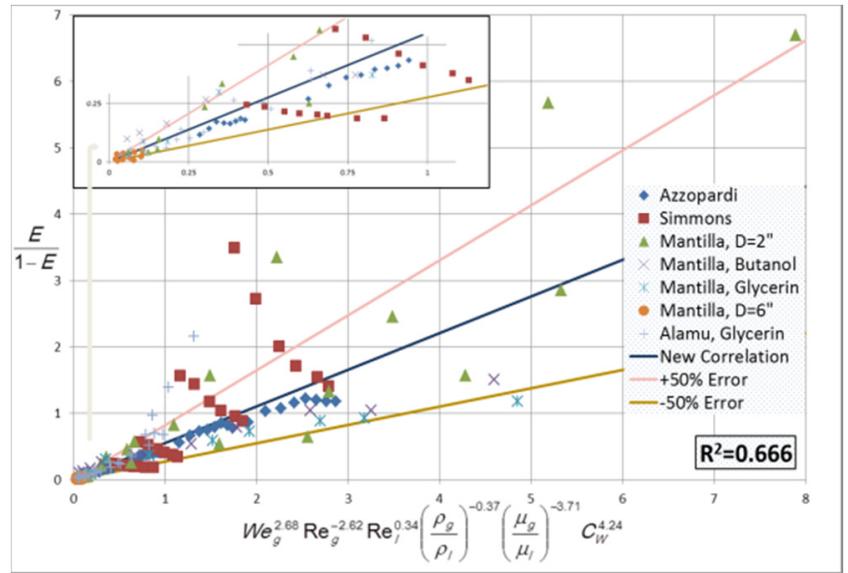

Figure 8: Fitting of the experimental data with the new entrained fraction correlation.

\section{Conclusions}

The characterization of the liquid film, the gas and the liquid-gas interface is mandatory for a thorough understanding of annular two-phase flow. This paper present a description of them and it also present a development of several new correlations for its more accurate description.

Regarding to the liquid film thickness, it has been observed that this variable decreases with superficial gas velocity, even though it becomes smaller for higher gas velocities. While the liquid film thickness increases with superficial liquid velocity. Concerning to wave celerity, an increase of this magnitude has been found for both velocities, gas and liquid, but with a higher influence of gas velocity. While wave frequency is strongly influenced by gas and liquid superficial velocities

Both magnitudes, the droplets size and their total amount, are mainly dominated by the gas superficial velocity, being less important the liquid velocity. An increase in gas flow rate results in a decrease in droplet sizes and vice versa (as gas velocity increases the droplets size distribution is shifted toward smaller diameters). Their mean velocity tend to be around $80 \%$ of the gas velocity. The entrained fraction present a common consistent trend in almost all works that have been found in the open literature, this magnitude is mostly dominated by the gas superficial velocity, being less important the effect of the liquid velocity. Higher values of gas and liquid velocities conduct to higher entrained fraction. 
Finish highlighting the improvement achieved with the new correlations developed in this work with respect to those found in the open literature.

\section{Acknowledgement}

The authors are indebted to the National Plan of I + D by the support of the project MODEXFLAT ENE-2013-48565-C02-01.

\section{References}

[1] Schubring D. \& Shedd T.A. "Wave behaviour in horizontal annular airwater flow". International Journal of Multiphase Flow, Vol. 34, pp. 636646, 2008.

[2] Alekseenko S., Antipin V., Cherdantsev A., Kharlamov S. \& Markovich D. "Two-Wave Structure of Liquid Film and Wave Interrelation in Annular Gas-Liquid Flow with and without Entrainment". Physics of Fluids, Vol. 21 N$^{\circ}$, Ner 061701, 2009.

[3] Crowe C.T. "Multiphase Flow Handbook (Mechanical Engineering)". CRC Press, Taylor \& Francis Group, 2006.

[4] Ishii M. \& Grolmes M.A. "Inception Criteria for Droplet Entrainment in Two-Phase Concurrent Film Flow". AIChE Journal, Vol. 21 pp. 308-318, 1975.

[5] Okawa T., Kotani A., Kataoka I. \& Naito M. "Prediction of Critical Heat Flux in Annular Flow Using a Film Flow Model". Journal of Nuclear Science and Technology, Vol. 40, pp. 388-396, 2003.

[6] Owen D.G. \& Hewitt G.F. "An Improved Annular Two-Phase Flow Model". Proceedings of the 3rd International Conference on Multiphase Flow, Hague, Netherlands, 1987.

[7] Sawant P., Ishii M. \& Mori M. "Prediction of Amount of Entrained Droplets in Vertical Annular Two-phase Flow". International Journal of Heat and Fluid Flow, Vol. 30, pp. 715-728, 2009.

[8] Fukano T. \& Furukawa T. "Prediction of the Effects of Liquid Viscosity on Interfacial Shear Stress and Frictional Pressure Drop in Vertical Upward Gas-Liquid Annular Flow”. International Journal of Multiphase Flow, Vol. 24, No 4, pp. 587-603, 1998.

[9] Tatterson D.F., Dallman J.C. and Hanratty T.J. "Drop sizes in annular gasliquid flows". AIChE Journal. Vol. 23, pp. 68-76, 1977.

[10] Paras, S.V. \& Karabelas A.J. "Properties of the Liquid Layer in Horizontal Annular Flow". International Journal of Multiphase Flow, Vol. 17, No 4, pp. 439-454, 1991.

[11] Schubring D. "Behaviour interrelationships in annular flow". PhD. Thesis University of Wisconsin-Madison, 2009.

[12] Alamu M. B. "Investigation of Periodic Structures in Gas-Liquid Flow". PhD. Thesis University of Nottingham, 2010.

[13] Mantilla I. "Mechanistic Modelling of Liquid Entrainment in Gas in Horizontal Pipes". PhD. Thesis University of Tulsa, 2008. 
[14] Al-Sarkhi A., Sarica C. \& Magrini K. "Inclination Effects of Wave Characteristics in Annular Gas-Liquid Flows". AIChE Journal, Vol. 58, pp. 1018-1029, 2012.

[15] Kolev N.I. "Multiphase flow dynamics: thermal and mechanical interactions". Springer Science \& Business Media, 3th Edition, 2007.

[16] Paras, S.V. \& Karabelas A.J. "Properties of the Liquid Layer in Horizontal Annular Flow". International Journal of Multiphase Flow, Vol. 17, No 4, pp. 439-454, 1991.

[17] Simmons M.J.H. \& Hanratty T.J. "Droplet size measurements in horizontal annular gas-liquid flow". International Journal of Multiphase Flow, Vol. 27, pp. 861-883, 2001.

[18] Al-Sarkhi A. \& Hanratty T.J. "Effect of pipe diameter on the drop size in a horizontal annular gas-liquid flow". International Journal of Multiphase Flow, Vol. 28, pp. 1617-1629, 2002.

[19] Lopes J.C.B. "Droplet sizes, dynamics and deposition in vertical annular flow". PhD. Thesis University of Houston, 1984.

[20] Fore L. B. \& Dukler A. E. "The distribution of drop size and velocity in gas-liquid annular flow". Int. J. Multiphase Flow 21, pp. 137-149, 1995.

[21] Fore L.B, Ibrahim B.B. \& Beus S.G. "Visual measurements of droplets size in gas-liquid annular flow". International Journal of Multiphase Flow, Vol. 28, pp. 1895-1910, 2002.

[22] Azzopardi B. J. "Drops in annular two-phase flow". International Journal of Heat Multiphase Flow, Vol. 23, pp. 1-53, 1997.

[23] Azzopardi B. J., Piearcey A. \& Jepson D. M. "Drop size measurements for annular two-phase flow in a $20 \mathrm{~mm}$ diameter vertical tube". Exp. Fluids, Vol. 11, pp. 191-197, 1991. 